\title{
Tubularized Penile-Flap Urethroplasty Using a Fasciocutaneous Random Pedicled Flap for Recurrent Anterior Urethral Stricture
}

\author{
Yong Jig Lee, Byung Kwon Lee \\ Department of Plastic and Reconstructive Surgery, Daegu Catholic University Medical Center, Catholic University of Daegu School of \\ Medicine, Daegu, Korea
}

This report describes the use of a tubularized random flap for the curative treatment of recurrent anterior urethral stricture. Under the condition of pendulous lithotomy and suprapubic cystostomy, the urethral stricture was removed via a midline ventral penile incision followed by elevation of the flap and insertion of an 18-Fr catheter. Subcutaneous buried interrupted sutures were used to reapproximate the waterproof tubularized neourethra and to coapt with the neourethra and each stump of the urethra, first proximally and then distally. The defect of the penile shaft was covered by advancement of the surrounding scrotal flap. The indwelling catheter was maintained for 21 days. A 9 month postoperative cystoscopy showed no flap necrosis, no mechanical stricture, and no hair growth on the lumen of the neourethra. The patient showed no voiding discomfort 6 months after the operation. The advantages of this procedure are the lack of need for microsurgery, shortening of admission, the use of only spinal anesthesia (no general anesthesia), and a relatively short operative time. The tubularized unilateral penile fasciocutaneous flap should be considered an option for initial flap urethroplasty as a curative technique.

Keywords Urethra / Penis / Urethral stricture / Recurrence / Surgical flaps
Correspondence: Yong Jig Lee Department of Plastic and Reconstructive Surgery, Daegu Catholic University Medical Center, Catholic University of Daegu School of Medicine, 33 Duryugongwon-ro 17-gil, Nam-gu, Daegu 705-718, Korea Tel: $+82-53-650-4578$

Fax: +82-53-650-4584

E-mail: handlee266@gmail.com

No potential conflict of interest relevant to this article was reported.

Received: 23 Nov 2011 • Revised: 14 Mar 2012 • Accepted: 16 Apr 2012

pISSN: 2234-6163 • elSSN: 2234-6171 • http://dx.doi.org/10.5999/aps.2012.39.3.257• Arch Plast Surg 2012;39:257-260

\section{INTRODUCTION}

Fasciocutaneous-flap urethroplasty has been known to produce positive outcomes in anterior urethral stricture [1]. The fasciocutaneous flap in non-Asian males provides an ample length (usually 13 to $15 \mathrm{~cm}$ ) of nonhirsute, well-vascularized tissue that can be used throughout the urethra [2]. However, the mean flaccid penile length in young Korean males is $6.9 \mathrm{~cm}$ (range, 6.1 to 7.7 cm) [3], and hair growth in the urethra results in infection and stone formation, which further predispose the area to recurrent stricture [4]. It is also known that penile skin flaps do not per- form well when they are tubularized in a single-stage procedure.

This article presents a case report of reconstructive tubularization with a one-stage unilateral penile fasciocutaneous flap of 5 $\mathrm{cm}$ in an Asian male after removal of the urethral stricture.

\section{IDEA}

A 33-year-old nonsmoking male presented with a 1-month history of difficulty in voiding his bladder. His cystourethrogram showed recurrence of a $2.5-\mathrm{cm}$ anterior urethral stricture (Fig. 1). This stricture had first been operated on about 8 years prior using 
graft urethroplasty. Removal of the narrowed pendulous urethral stricture was performed using the urethrotome and repair of the cut urethra was performed using the end-to-end anastomosis method. About 7 years later, he began occasionally suffering from difficulty voiding his bladder and received three more direct vision internal urethrotomies over a 2-year period.

The patient was scheduled for a single-stage suprapubic cystostomy procedure. He was placed in a supine position for pendulous lithotomy. After inserting an 18-Fr catheter to ensure the correct

\section{Fig. 1. Preoperative cystourethrogram}

Urethrography through suprapubic cystostomy and the external meatus revealed a $2.5-\mathrm{cm}$ functional gap at the penile urethral level.

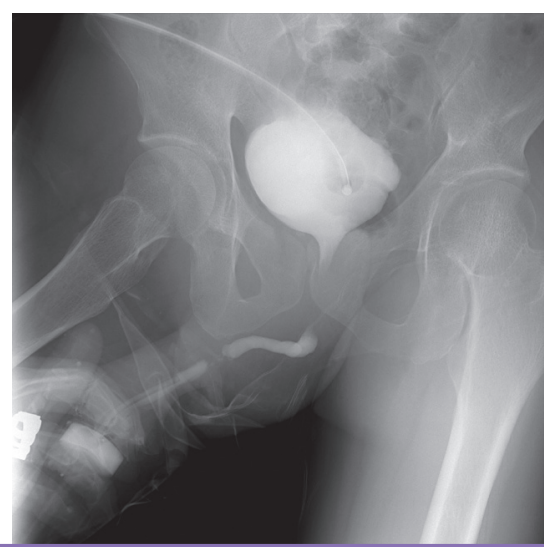

anatomical position of the penis, exposing the penile stricture, and excising the correct stricture, including more proximal and distal scar tissue in the functional stricture shown in the cystourethrogram, the flap width was measured and outlined to $2.5 \mathrm{~cm}$. The flap length was then measured at $5 \mathrm{~cm}$ for replacement of the gap length while the flap was temporarily wrapped around the catheter. Only a single ventral penile incision line was needed for resection of the stricture and for flap elevation. Electrocauterization was applied directly to the hair follicles. If the hair could be pulled from the follicle without resistance, the cautery was deemed successful.

Subcutaneous buried interrupted 5-0 Vicryl sutures were used to reapproximate the luminal surface of the waterproof tubularized neourethra and to coapt the neourethra and each stump of the urethra, first proximally and then distally (Fig. 2). Swelling increased gradually for 4 days after the operation, and then subsided. He was discharged on the ninth postoperative day. A 4-month postoperative cystoscopy showed no flap necrosis, no mechanical stricture, and no hair growth on the lumen of the neourethra (Fig. 3). The patient showed no voiding discomfort 9 months after the operation.

\section{DISCUSSION}

Trauma is likely to be the most common cause of anterior stric-

\section{Fig. 2. Intraoperative and postoperative photographs}

(A) Illustration shows a midventral incision, removal of the stricture, the elevation of the fasciocutaneous random skin flap, and formation of the neourethra by tubularization. (B) A transverse fasciocutaneous random flap was harvested with the pedicle of the tunica dartos fascia as a conduit and wrapped around an 18-Fr catheter with four 3-0 Vicryl stay sutures before being buried subcutaneously with 5-0 Vicryl sutures. (C) Buried sutures were used for tubularization and end-to-end anastomosis between the neourethra and each side of the remaining distal and proximal urethral stumps. The catheter was reinserted through the external meatus into the bladder by inflating the indwelling catheter. The defect of the penile shaft was covered with the advancement of the surrounding scrotal flap. (D) The defect of the donor site of the penile flap was covered with the advancement of the scrotal flap. (E) On the eighteenth postoperative day, there was no sign of flap necrosis, dehiscence, or infection.
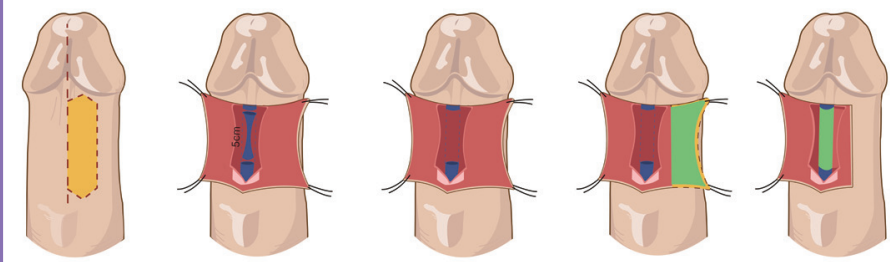

(A)
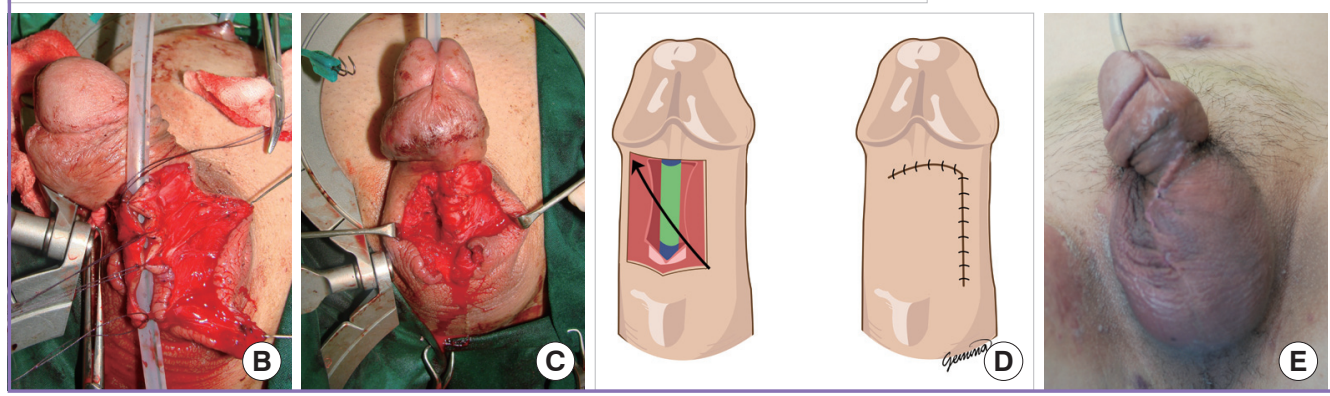


\section{Fig. 3. Four-month postoperative cystoscopy}

Postoperative cystoscopy image showing no flap necrosis or hair growth on the lumen of the neourethra.

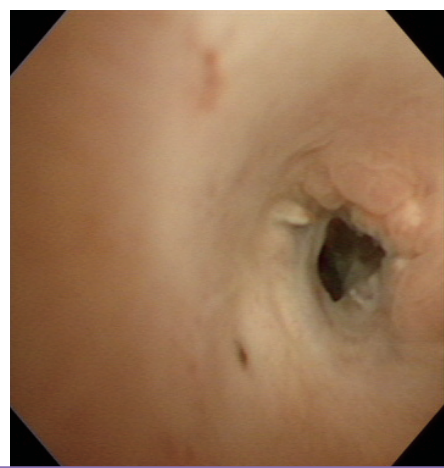

tures in virtually all populations. Instrumentation strictures are usually due to catheterization and typically located at the penobulbar junction, and most are ischemic in origin [5].

The male urethra can be divided anatomically into its anterior and posterior segments. The anterior portion is further divided into the bulbous, pendulous (penile), and fossa navicularis or meatal urethral segments (also called the distal urethra). The bulbar urethra is enclosed by the bulbospongiosus muscle, and the penile urethra runs from the distal margin of the bulbospongiosus to the fossa navicularis and external meatus. The posterior urethra is the section of tissue between the bladder neck and the bulbar urethra, and includes the bladder neck proper, the prostatic urethra, and the membranous urethra surrounded by the external urethral sphincter mechanism [2].

The 'reconstructive ladder' principle, in which a progression from simple to complex surgeries is only considered after the failure of several successive interventions, has been applied to the treatment of urethral stricture for some time. However, the development of refined reconstructive techniques enables curative treatments of even complex strictures [6].

Because the penile skin flap has its own blood supply, mobilized tissue can be used to fill the gap left after excising a stricture without requiring a graft bed. The use of a circular penile fasciocutaneous skin flap was associated with a recurrence rate of 50\% in patients with strictures due to balanitis xerotica obliterans, whereas all patients with strictures caused by other etiologies had excellent results $[2,7]$. Nearly all patients, including those who have been circumcised, will have adequate preputial redundancy to permit a circumferential flap of up to $2.5 \mathrm{~cm}$ to be harvested without compromising primary closure or the functional results [2]. A fasciocutaneous flap is especially suitable for strictures longer than $5 \mathrm{~cm}$, corresponding to more than half of the entire penile length [1].

The corpora cavernosa and corpus spongiosum are invested by both a deep fascial layer (Buck's fascia and penis fascia) and a superficial layer of loose areolar tissue known as the dartos fascia. The circular penile fasciocutaneous flap uses the tunica dartos fascia as a conduit to contain the vascular pedicle of the deep dorsal vein, and dorsal (dorsolateral and ventrolateral) arteries from the superficial and deep external pudendal arteries of the femoral artery [2]. The term 'fasciocutaneous flap' refers to the use of Buck's fascia as the primary supporting fascia of the tunica dartos vascular pedicle as it passes to the island of penile skin [7]. The superficial dartos fascia is a thin, membranous layer of loose subdermal tissue devoid of fat that lies immediately beneath the skin. This fascia must be attached to penile skin in order to preserve the delicate subdermal plexus and prevent skin necrosis. A layer of areolar tissue moves freely over Buck's fascia, is devoid of fat, and is continuous with the membranous layer (Scarpa's fascia) of the fascia of the anterior abdominal wall, the tunica dartos of the scrotum, and Colles' fascia in the perineum [2].

Long-term follow-up of fasciocutaneous-flap urethroplasty applied to anterior urethral strictures show an excellent success rate of almost $94 \%$ [1], but the presence of comorbidities or a patient's personal preference may lead some patients to choose symptomatic management of their stricture disease (by periodic dilation and internal urethrotomy) over a potentially curative procedure such as urethroplasty [6].

The design of this transverse random flap, with the tunica dartos fascia as a conduit and based on the dorsal axial vascular pedicle, means that it can be harvested from the initial ventral midline incision used for exploration and resection of anterior urethral strictures. This can be performed on one side first, and in cases of recurrence, the procedure can be performed on the other side as well. This can be performed under spinal anesthesia and with a relatively short operative time because no microsurgical reconstruction is necessary.

The use of a penile flap provides the potential advantage of its elasticity resulting in less dehiscence during morning erections following the operation. This inherent elasticity also decreases the overall width of the flap. The 18-Fr catheter inserted is $6 \mathrm{~mm}$ in diameter (corresponding to the French size divided by 3 ), based on $\Pi(3.14) \times(\text { radius })^{2}$, means that the width of the tubularized flap should be $28 \mathrm{~mm}$ (the actual size of the flap in our patient was $25 \times 50 \mathrm{~mm}$ ). There is a small diverticulum at the proximal anastomosis site resulting from flap elasticity, but the site may provide for laxity of the neourethra during penile erection. In addition, electrical coagulation can be used to prevent diverticulitis resulting from remnant hair follicles.

We highlight the main differences of our design from other methods $[2,7,8]$, which include one longitudinal incision line of the neourethra and whole neourethral reconstruction after 
extirpation of the structural stricture.

Compared with other methods, the transverse flap is easier to use than the axial flap in creating the tubular shape. The transverse flap needs just one single longitudinal incision line for the neourethra, and the axial flap requires the skin flap to be split for tubularization [8]. Fewer incision lines could reduce the complications of the neourethra such as fistula and dehiscence, among others.

The ventral onlay flap is the removal of the part of the urethral stricture, release, and insetting the flap [2,7]. However, our method involved the removal of not only the anatomical but also the functional urethral stricture.

In conclusion, the transverse tubularized penile fasciocutaneous flap should be considered an option in the curative treatment of recurrent or naïve long anterior urethral strictures.

\section{REFERENCES}

1. Schwentner C, Seibold J, Colleselli D, et al. Anterior urethral reconstruction using the circular fasciocutaneous flap tech- nique: long-term follow-up. World J Urol 2011;29:115-20.

2. Carney KJ, McAninch JW. Penile circular fasciocutaneous flaps to reconstruct complex anterior urethral strictures. Urol Clin North Am 2002;29:397-409.

3. Son H, Lee H, Huh JS, et al. Studies on self-esteem of penile size in young Korean military men. Asian J Androl 2003;5: 185-9.

4. Somerville JJ, Adeyemi OA, Clark PB. Long-term results of two-stage urethroplasty. Br J Urol 1985;57:742-5.

5. Andrich DE, Mundy AR. What is the best technique for urethroplasty? Eur Urol 2008;54:1031-41.

6. Tonkin JB, Jordan GH. Management of distal anterior urethral strictures. Nat Rev Urol 2009;6:533-8.

7. Buckley J, McAninch J. Distal penile circular fasciocutaneous flap for complex anterior urethral strictures. BJU Int 2007; 100:221-31.

8. McAninch JW, Morey AF. Penile circular fasciocutaneous skin flap in 1-stage reconstruction of complex anterior urethral strictures. J Urol 1998;159:1209-13. 